\title{
Toward an Interlocal Collaboration Decision Model of Local Government in China
}

\author{
Liming Suo \\ University of Electronic Science and Technology of \\ China \\ Chengdu, China \\ e-mail:dinastysuo@126.com
}

\author{
Yudi Yuan \\ University of Electronic Science and Technology of \\ China \\ Chengdu, China \\ e-mail: yudiyuancathy@ sina.com
}

\begin{abstract}
In the context of economic development and regional integrated development, faced with action challenges and 'economic' governance; it is essential to figure out how to achieve sustainable development of interlocal collaboration in order to attain the optimal interlocal collaboration in governance decision-making. Optimal collaboration decision model is based on the collaborative willingness and interlocal collaboration transaction cost. It is vital to find the best model under different conditions, so that it would be possible to change current institution and ill policy environment.
\end{abstract}

Keywords-Collaboration; Willingness; Transaction Costs,; Interlocal Collaboration; Model

\section{INTRODUCTION}

Since the 1990s it has been a significant tendency that the government is prone to achieving sustainable development in interlocal collaboration, in the context of flourishing economic development of Yangtze River Delta, Pearl River Delta, Beijing-Tianjin Delta and other economic regions. Recently, interlocal collaboration has, from the east, been expanded into the west gradually, and has been evolved into interlocal collaboration from adjacent areas, has been transferred into implementation by varied ways of collaboration from mere dependence on planning of the central government, and has been from simplified complementary-resource collaboration converted into comprehensive economic and social collaboration including infrastructure, environment, resources and tourism etc, with the implementation, projection and establishment of industrial inter-transfer of the East and West, 9+2 Pan Pearl River Delta, Changsha-Zhuzhou-Xiangtan City Group, Chengdu-Chongqing Economic Zone, Guanzhong-Tianshui Economic Zone etc, which have implemented in-depth development and gradual standardization of interlocal collaboration. As a result, it turns into one of principle functions and activities of local government to seek successful interlocal collaboration, implement efficient collaborative governance model and establish public policies that are suitable for interlocal collaboration.

In this context, intergovernmental relations, regional administration, regional coordination, interlocal collaboration and collaborative governance etc become top issues in the administration science. Scholars have analyzed a lot of cases of Yangtze River Delta (Zhao Feng, Jiang Debo, 2011[1]), Pearl River Delta(Wang Weiyi, 2010[2]), Beijing-Tianjin Economic Zone (Ma Hailong, 2010 [3]; Cui Jing, 2011[4]) and other economic regions to illustrate collaborative effects, collaborative organizational forms, coordination, collaboration forms etc of local government. Furthermore, on the foundation of conventional theory of intergovernmental relations, scholars have developed conceptions of regional administration and regional public administration etc to proceed standard research of establishment of administrative system. Chen Ruilian, Zhang Jingen (2002) [5] stimulated a pioneering exploration on general theory of regional administration and regional public administration. Shi Zulin, Liu Feng (2003) [6] proposed to study the innovation of regional public management in terms of regional study, regional culture and organization etc; Wang Jian, Bao Jing et al (2004) [7] and Wang Chuanlan (2006) [8] had a creational conception of evolvement of compound administration; Chen Ruilian (2005) [9] stated that it was necessary definitely for creation from conception of administrative division to governance of regional public administration, from bureaucracy to inter-network system etc. However, current researches remain in the aspects of conclusion of cases, establishment of systems etc, lacking of theoretical researches on models of collaboration and performance of local government. This research establishes an analytic model to analyze the collaboration of local government.

\section{THEORETICAL BACKGROUND}

Three research models of theory of western public administration exist (David E.McNabb, 2008[10]): Institutionalism Model, Behaviorism Model, and Rational Decision Theory Model. Theory of interlocal collaboration and administration is derived from those three research models.

Priviously, scholars primarily applied conventional public economy paradigm of public goods, externality and scale economy, with developing an analytic model of public administration. This analytic model is mainly applied to problems and solutions of macroscopic and medium regional public administration, such as protection of regional environment, regional sustainable development, watershed administration and other problems of resource environment. It is originated from the application of regional public goods. On the foundation of research of Tiebout (1956) [11], Teske and Schneider et al (1995) [12] argued the efficiency of dispersive local system, Basolo (2003) [13]proved that interlocal collaboration and concentration benefited local government on externality of management of public problems. Later, in the context of 
institutional framework of Ostrom (1990) [14] and analysis of group action by Olson (1995) [15],Krueger and McGuire (2005) [16], Feiock $(2004,2005,2007)$ [17,18,19] et al brought transaction costs into interlocal collaboration administration, and created Institutional Collective Action (ICA). Jin Taijun (2007) [20] stated that the obstruction of collaboration of government divisions was derived from huge transaction costs of path dependence brought by scale effects of local government, learning effects, coordination effects, adaptive anticipation and strategy effects, from the perspective of system analysis. As a result, the analytic perspective of institution which emphasizes on individuals making rational decisions becomes the tendency of interlocal collaboration, and constitutes the theoretical foundation of interlocal collaboration of local government.

\section{INTERLOCAL COLLABORATIVE ACTIONS OF LOCAL GOVERNMENT AND INFLUENTIAL ELEMENTS}

Based upon the foundation of model and premised assumptions, it is assured by the author that collaborative action decisions of local government are influenced by subjective finite reasons and the environment of external institutional organizations. These two perspectives represent collaborative willingness and transaction costs respectively.

\section{A. Collaborative willingness of local government and interlocal collaboration}

Decisions of local government can be influenced by individual subjective elements. Collaborative willingness is one of essential influential elements of interlocal collaborative actions. We divide collaborative willingness into two dimensions: preference intensity and preference structure. They constitute space and indifference curve of interlocal collaborative willingness of local governments. In Chart 2, horizontal and vertical coordinates represent two random collaborative fields for choice, for example, construction of road infrastructure, market collaboration, construction of Science and Technology Park, new region of real estate, and governance of water environment etc. Ordering differences exist in these fields for decision makers of local governments. However, decision makers could order them according to their preference, so that it can be included into local characteristics and highlights etc. Two collaborative fields could be composed into a platform, which is called collaborative willingness space, and internal coordinates represent different intensities of collaborative willingness. The character of intensity of collaborative willingness shows the willingness and action frequency of seeking collaborative partners of local government, including the grade and time of documents, frequency of essential speeches of officials, economic investment of interlocal collaboration, frequency of taking initiative to seek collaboration, and time of negotiation etc. In the space of collaborative willingness, different combination of collaborative fields could constitute specific collaborative planning. Results of collaborative planning could rely on the intensity of collaborative preference, and the more intensive it is, the more collaborative content it will be.
There are three indifference curves A, B, and C in Fig.1, and they represent different collaborative intensities. Each point on any curves represents different combination of collaborative fields. However, collaborative intensities on the same curve are of no difference. For example, combinations represented by each point of $\mathrm{A}, \mathrm{B}, \mathrm{C}$ have the same collaborative willingness, and represent those local governments with the same intensity of collaborative preference or actions. However, collaborative willingness of any points on Curve $B$ is always higher than that on Curve $\mathrm{A}$, and that on Curve $\mathrm{C}$ is higher than that on Curve $\mathrm{B}$ similarly.

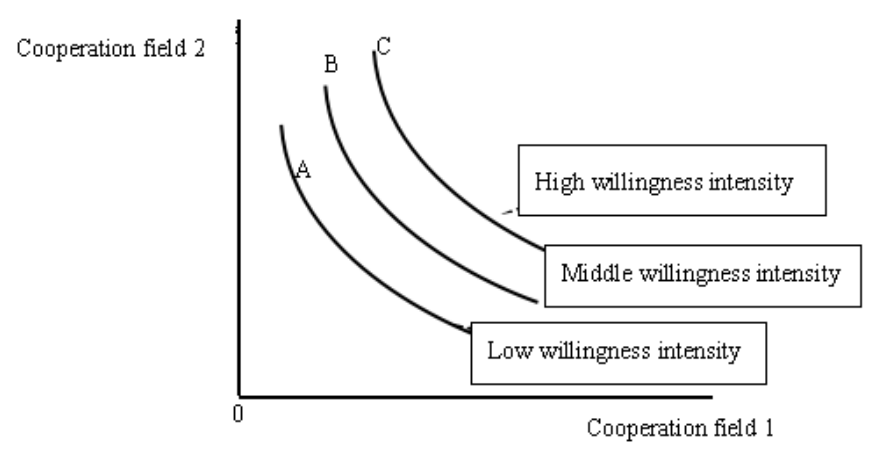

Figure 1. Space of collaborative willingness of local government

In addition, it is considered that the relationship between performance and collaborative willingness in interlocal collaboration does not increase linearly, but has an optimum degree, which is determined by local economic level, environmental conditions, development strategies, and institutional environment. Moreover, an optimum collaborative performance is based on the matching of exogenous environment and collaborative willingness of local government.

\section{B. Transaction costs of interlocal collaboration}

Rational choosing theory takes gregarious decision environment into relative analysis, and it must consider about how resolutive environment militates specific choice systematically, besides taking decision environment of local government when making gregarious decisions into consideration. Transaction costs could characterize system environment and collaborative dilemma of local government, as core conception of system analysis.

Inter-collaborative choice of local governments is a decision resulted from independent development of local governments and model of interlocal collaborative development. Local governments must consider about endogenous and exogenous transaction costs, when making decisions about whether independent development or interlocal collaborative development shall be chosen. On the basis of Coase (1937) [21] who regarded nature of enterprise as governance mechanism, Williamson (1989) [22] defined transaction costs as costs that were capable to 
be compared in the choice of valid governance mechanism, and as costs of planning, coordination and supervision which were needed when governments completed missions. Independent development and interlocal collaboration of local government are two governance structures to be chosen from. If transaction costs equal zero, the results would be the same by choosing one of these two kinds of mechanisms randomly, but Endogenetic Transaction Cost (NTC) of interlocal collaboration and Exogenous Transaction Costs (XTC) of independent development are a dilemma for the government. When governments supply public goods or during the economic development, if independent development is chosen, inter-collaborative costs with other governments are XTC, and the level of which is one of the base of judgment of collaborative choice. However, it will reduce fixed costs, average costs, and inter-market transaction costs of other local governments if interlocal collaboration is chosen. Otherwise, NTC will increase at the same time, that is, costs of Neo-management and Neo-coordinate resulted from intricate organization and relationship will increase. For local government, decisions between two development models constitute costs of choosing phase of collaborative strategies.

\section{INTERLOCAL COLLABORATIVE DECISION MODEL}

Interlocal collaborative decision model is an optimal decision model between independent development and interlocal collaboration. When a local government encounters possible collaborative opportunity, it needs to propose a decision among independent development, collaborative development and joining in a large-scale collaborative treaty. This kind of collaborative treaty includes all kinds of collaborative forms, including alliance, strategic partners, regional protocol, economic zone, city group, and collaborative zone etc, and they represent scale and organization of regional governance. For example, independent development is the minimum regional governance scale. Regional scale has a direct influence on changes of endogenous and exogenous transaction costs of individual local government. The endogenous transaction costs, originated from internal coordination and management, of individual local government increase with the expansion of regional governance scale. However, its exogenous transaction costs, originated from market transaction, decrease. Local government seeks balance between those two transaction costs. The ultimate equilibrium state is the regional scale when exogenous and endogenous transaction costs reach equalization.

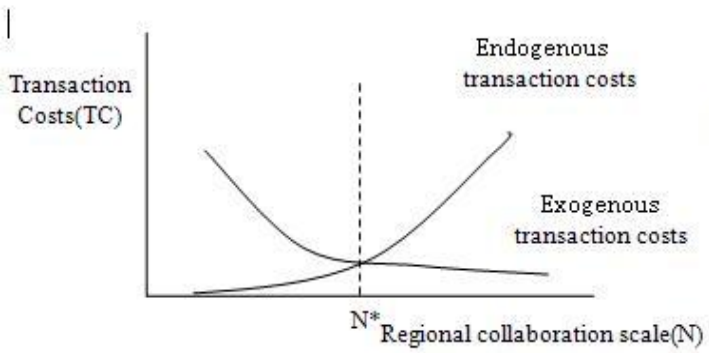

Figure 2. Collaborative Choice And Analysis Model Of Local Governments In Interlocal Collaboration

In Fig.2, the horizontal axis represents interlocal collaborative scale $(\mathrm{N})$. It consists of the number of participants, and four elements--sum of money, content of collaboration treaty and the number of participated departments. As there is a positive correlation between the last three elements and the number of participants, it will use the number of participants to represent collaborative scale, denoted by the countable $\mathrm{N}$. Vertical axis represents transaction costs (TC), and two curves in Fig.2 are exogenous and endogenous transaction costs respectively. Endogenous transaction costs increase with the increase of the number of participants. Exogenous transaction costs result from competition and market transaction. If a local government chooses independent development, exogenous transaction costs are high, but could be internalized with the increasing number of participants, so that exogenous transaction costs could be decreased.

When a local government is pending on whether to participate in an interlocal collaboration proposal or not, which is composed of $\mathrm{N}-1$ members, it will make a comparison between endogenous and exogenous transaction costs. If endogenous transaction costs are greater than exogenous transaction costs, it states that costs of coordinate of interlocal collaboration scale are at an excessively high level, and the scale must be decreased; in contrast, if exogenous transaction costs are greater than endogenous transaction costs, it states that market transaction costs from interlocal collaboration scale are excessively high, and the scale needs to be expanded. Thus, if exogenous transaction costs are equal to endogenous transaction costs, the adjustment led by local governments for interlocal collaboration scale is at the state of equalization. When $\mathrm{N}^{*}=1$, it states that local governments do not need collaboration with other governments any more, and it is the top priority for interlocal collaborative analysis.

\section{CONCLUSIONS}

Firstly, interlocal collaboration refers to a series of institutional arrangements that the central or local governments start from their rational decisions, change collaboration willingness by constructing formal and informal collaboration protocol and incentive mechanism among government subjects, form group actions, optimize collaboration mode, and gradually form optimal 
collaboration structure which could decrease transaction costs and realize Pareto Improvement.

Secondly, it regards motivation, preference and actions of individual local government as individual variables which can influence interlocal collaboration, and regards interrelation of multiple local governments and possible transaction costs as structure variables. This kind of analysis paradigm reveals the conflict between individuals and group rationality, and possible structure after interaction. However, combined action of collaboration willingness and transaction costs with different levels can have an impact on actions and performance of interlocal collaboration, and different participants could present different interlocal collaboration conditions.

Thirdly, for collaboration willingness, the key of interlocal collaboration coordination is adjustment of preference intensity and structure of interlocal collaboration. However, local governments should pay attention to whether collaboration willingness of potential partners is compatible with collaboration demand, so that they can lessen the loss of collaboration performance resulted from subjective factors. In further study, empirical analysis can be made for collaboration willingness and influential elements of local government officials in different regions and at different levels.

Finally, for transaction costs, the key of interlocal collaboration coordination is to decrease obstacles of interlocal collaboration. On the one hand, superior governments need to decrease inter-collaboration obstacles of subordinate governments in jurisdiction, or decrease transaction costs resulted from information and negotiation by creating platform of collaboration policy. On the other hand, participated governments should establish efficient and available supervision and incentive constraint mechanism for proceeding collaboration projects, and illustrate explicitly the subjects, objects, and relevant functions, regulations etc of inter-governance, in order to decrease transaction costs which may be produced from execution and agency. In further study, it can propel empirical analysis of transaction costs and influential elements in different forms of interlocal collaboration.

\section{ACKNOWLEDGMENT}

This paper is supported by Nation Science Funding(71003013); "Fundamental Research Funds for the Central Universities"(ZYGX2010X024), Sichuan Province Cyclic Economy Research Center Project(XHJJ-0914)

\section{REFERENCES}

[1] Zhao Feng, Jiang Debo. Experience Reference and Plan of Further Development of Collaborative Mechanism in Yangtze River Delta [J]. Public Administration of China, 2011.2: 81-84
[2] Wang Weiyi, Difficulties and Opportunities of Collaboration of Pan-Pearl River Delta Region in New Situations [J]. Asian Pacific Economy. 2010 (6): 16-120

[3] Ma Hailong. Model Decision of Governance in Beijing-Tianjin-Hebei Region [J]. Journal of Beijing Administrative College. 2010 (6): 7-11

[4] Cui Jing. Model Research of Local Governments Coordination in Metropolitan Areas of Beijing-Tianjin-Hebei Region with Perspective of Holistic Governance [J]. Beijing Society Science, 2011 (2): 34-37

[5] Chen Ruilian, Zhang Jingen. Coordination of Inter-relationship of Governments in the Regional Economic Development [J]. Administrative Management of China. 2002 (12): 65-68

[6] Shi Zulin, Liu Feng. Innovation of Regional Public Administration in New Period [J]. Population Resources and Environment of China. 2003 (3) : 16-19

[7] Wang Jian, Bao Jing, Liu Xiaokang, Wang Dianli. Proposal of Compound Administration--New Idea of Resolution of Conflicts of Regional Economic Integrity and Administrative Division in Contemporary China [J]. Administrative Management of China, 2004 (3) : 44-48

[8] Wang Chuanlan. Multi-Component Administrative System: Construction of Realization of Regional Administration [J]. Social Science, 2006 (4) : 111-118

[9] Chen Ruilian, Liu Yaping. Collaboration and Innovation of Governments in Pan-Pearl River Delta Region [J]. Academic Research. 2007 (1): 42-50

[10] David E. McNabb. Research Methods in Public Administration and Nonprofit Management $\left(2^{\text {nd }}\right)$ [M]. M.E. Sharpe Press.London.2008

[11] Tiebout, C. A Pure Theory of Local Expenditures. Journal of Political Economy [J], 64, 1956:416-424.

[12] Teske, P., Schneider, M., Mintrom, M., \& Best, S. The Empirical Evidence for Citizen Information and A Local Market for Public Goods: Response. American Political Science Review [J], $1995.89(3): 707-709$.

[13] Basolo. V. U.S. Regionalism and Rationality [J]. Urban Studies.2003.40:447-462.

[14] Ostrom, Elinor. Understanding Institutional Diversity [M]. Princeton. Princeton University Press. 2005

[15] Mancur Lloyd Olson, Jr,. Logic of Collective Action [M]. Sanlian Bookstore LTD Shanghai Branch. Shanghai People's Publishing House. 1995

[16] Krueger, Skip and McGuire, Michael, "A Transaction Costs Explanation of Interlocal Government Collaboration". Working Group on Interlocal Services Collaboration. 2005: 21

[17] Feiock, Richard C. A Quasi-Market Framework for Development Competition[J]. Journal of Urban Affairs. 2002.24(2): 123-42.

[18] [18] Feiock, Richard C. Metropolitan Governance: Conflict, Competition and Collaboration [M]. Washington D.C.: Georgetown University Press. 2004

[19] Feiock, Richard C. Rational Decision and Regional Governance [J], Journal of Urban Affairs, 2007. 29 (1): 47-63.

[20] Chen Guoquan, Li Yuanlin. Interlocal Collaboration in Integrity of Yangtze River Delta [J]. Jianghai Academic Journal. 2004 (5) : 92-98

[21] Coase, Ronald H. The Nature of Firm. Economica. N.S. 1960 (3): $1-44$

[22] Williamson, Oliver E. Transaction Cost Economics. In Handbook of Industrial Organization [C], 1989. Volume 1, edited by $\mathrm{R}$. Schmalensee and R. Willig. Amsterdam: North-Holland 